\title{
Alain Billionnet \\ Une nouvelle méthode pour le problème de partitionnement fondée sur une évaluation par excès de la solution
}

Revue française d'automatique, d'informatique et de recherche opérationnelle. Recherche opérationnelle, tome 15, n 2 (1981), p. $139-152$.

<http://www.numdam.org/item?id=RO_1981_15_2_139_0>

(C) AFCET, 1981, tous droits réservés.

L'accès aux archives de la revue « Revue française d'automatique, d'informatique et de recherche opérationnelle. Recherche opérationnelle » implique l'accord avec les conditions générales d'utilisation (http://www.numdam.org/ legal.php). Toute utilisation commerciale ou impression systématique est constitutive d'une infraction pénale. Toute copie ou impression de ce fichier doit contenir la présente mention de copyright.

\section{Numdam}

Article numérisé dans le cadre du programme

Numérisation de documents anciens mathématiques

http://www.numdam.org/ 


\title{
UNE NOUVELLE MÉTHODE POUR LE PROBLĖME DE PARTITIONNEMENT FONDÉE SUR UNE ÉVALUATION PAR EXCÈS DE LA SOLUTION (*)
}

\author{
par Alain Billionnet $\left({ }^{1}\right)$
}

Résumé. - Nous présentons d'abord deux heuristiques pour déterminer une bonne évaluation par excès du problème de partitionnement. Ces heuristiques, fondées sur la recherche d'un ensemble stable de poids maximal dans un graphe, donnent souvent, pour des exemples de taille moyenne, la solution optimale du problème. Nous montrons, ensuite, comment la comparaison de cette évaluation par excès et d'une expression réduite de la fonction économique permet de simplifier le problème de partitionnement considéré. Cette méthode est appliquée à plusieurs exemples. Nous montrons, enfin, comment certaines réductions de la fonction économique permettent d'améliorer la méthode et d'obtenir une très bonne évaluation par défaut de la solution.

Mots clés : Problème de partitionnement, graphe, ensemble stable.

Abstract. - First two heuristic methods are introduced in order to obtain a good upper bound for the solution of the set partitioning problem. These heuristic methods based upon the search of a maximum weighted stable set in a graph often provide the optimal solution for examples of average size. Then we show how the comparison between this upper bound and a reduced expression of the economical function enables the set partitioning problem to be simplified. This method has been applied to several examples. It is shown at last how some reductions of the economical function allow to improve the method and to obtain a very good lower bound for the solution.

Keywords: Set partitioning problem, graph, stable set.

\section{INTRODUCTION}

Le problème de partitionnement $\mathrm{PP}$ :

$$
\text { minimiser } z=c x \text {, }
$$

avec

$$
\left.\begin{array}{c}
A x=e, \\
x_{j}=0 \text { ou } 1,
\end{array}\right\}
$$

$\left({ }^{*}\right)$ Reçu octobre 1979.

(') Institut Informatique d'Entreprise, C.N.A.M., 292, rue Saint-Martin, 75141 Paris Cedex 03.

R.A.I.R.O. Recherche opérationnelle/Operations Research, 0399-0559/1981/139/\$5.00

(C) AFCET-Bordas-Dunod 
où $A=\left(a_{i j}\right)$ est une $m \times n$ matrice donnée et telle que $a_{i j}=0$ ou $1 ; e$, un $m \times 1$ vecteur dont tous les éléments sont égaux à $1 ; c$, un $1 \times n$ vecteur à coefficients positifs connus; $x$, le vecteur $n \times 1$ des inconnues $x_{j}$; est un problème très contraint qui se résout bien par énumération implicite $[10,6]$. Cependant ces auteurs développent d'importantes arborescences. Plusieurs techniques ont été utilisées pour réduire la taille de ces arborescences; elles consistent, en général, à calculer une bonne évaluation par défaut de la solution. [8] et [11] utilisent la solution du programme linéaire continu associé au problème $\mathrm{PP}$; [7] réduisent la fonction économique à partir d'une combinaison linéaire des contraintes ce qui leur permet de définir une évaluation par défaut et des coûts réduits pour chaque variable sans avoir à résoudre de programme linéaire.

Nous présentons, ici, une nouvelle méthode fondée sur une bonne évaluation par excès de la solution. Pour tous les exemples que nous avons examinés cette méthode permet de déterminer la solution du problème $\mathrm{PP}$ sans recourir ni à une procédure arborescente ni à la programmation linéaire. Dans les cas où elle ne conduit pas à la solution de PP, elle permet de réduire notablement la taille du problème.

Les principales caractéristiques de cette méthode sont :

(a) la fonction économique est réduite à partir d'une combinaison linéaire des contraintes comme dans l'algorithme de Gondran et Laurière (\$2);

(b) il est difficile, pour le problème de partitionnement, de calculer une bonne évaluation par excès de la solution. Une heuristique appliquée à la recherche d'un ensemble stable de poids maximal dans un graphe permet de déterminer cette évaluation ( $\$ 4)$;

(c) toutes les implications sont utilisées systématiquement : d'une part, les implications obtenues en confrontant l'expression de la fonction économique réduite et l'évaluation par excès de la solution, d'autre part, les implications classiques dues aux contraintes (1) (\$5);

(d) enfin, dans le paragraphe 7 , nous montrons comment la méthode peut être améliorée en essayant d'augmenter, au maximum, les coûts réduits de chaque variable.

\section{RÉDUCTION DE LA FONCTION ÉCONOMIQUE}

Soit :

$$
J_{i}=\left\{j \mid a_{i j}=1\right\} \quad \text { et } \quad c_{j_{o}}=\min _{j \in J_{i}} c_{j} .
$$

Puisque :

$$
\forall i \in\{1, \ldots, m\}, \quad \sum_{j=1}^{n} a_{i j} x_{j}=1,
$$

R.A.I.R.O. Recherche opérationnelle/Operations Research 
la fonction économique peut s'écrire :

$$
z=c_{j_{0}}+\sum_{j=1}^{n} c_{j}^{\prime} x_{j}
$$

avec $c_{j}^{\prime}=c_{j}-a_{i j} c_{j_{0}}$.

En procédant ainsi avec chacune des $m$ contraintes, on obtient une fonction économique de la forme :

$$
z=z_{d}+\sum_{j=1}^{n} c_{j}^{\prime} x_{j}
$$

avec $z_{d}$ et $c_{j}^{\prime} \geqq 0$.

Nous retiendrons les critères proposés par [7] pour déterminer l'ordre dans lequel les contraintes sont utilisées :

Critère 1, choisir la contrainte qui comporte le moins de variables;

Critère 2, à chaque contrainte associer le nombre de variables ayant le plus petit coût. Choisir celle pour laquelle ce nombre est minimal;

Critère 3, à chaque contrainte associer le plus petit coût parmi les variables de cette contrainte. Choisir celle pour laquelle ce coût est le plus grand.

\section{GRAPHE ASSOCIÉ AU PROBLÈME DE PARTITIONNEMENT}

Nous avons montré dans [1] que tout problème de partitionnement. avant une solution peut se poser comme la détermination d'un ensemble stable de poids maximal dans un graphe simple, $G=(X, V)$, défini de la façon suivante :

$$
X=\left\{x_{1}, \ldots, x_{n}\right\} ;
$$

$\left[x_{r}, x_{s}\right]$ est une arête de $G$ si et seulement si les variables $x_{r}$ et $x_{s}$ apparaissent ensemble dans au moins une des contraintes:

$$
\left[x_{r}, x_{s}\right] \in V \Leftrightarrow \exists i \in\{1, \ldots, m\} \quad \text { tel que } a_{i r} . a_{i s}=1 ;
$$

le « poids » de chaque sommet est :

$$
p\left(x_{j}\right)=L \cdot \sum_{i=1}^{m} a_{i j}-c_{j} \quad \text { avec } \quad L>\sum_{j=1}^{n} c_{j} .
$$

Le poids d'un ensemble stable, $S$, est noté $P(S)$.

ReMARQue 1 : Si le graphe $G$ admet $k$ composantes connexes on recherchera, pour déterminer un ensemble stable de poids maximal de $G$, un ensemble stable vol. $15, \mathrm{n}^{\circ} 2$, mai 1981 
de poids maximal dans chacune des $k$ composantes. Cela signifie que le problème $\mathrm{PP}$ se décompose en $k$ sous-problèmes $\mathrm{PP}_{1}, \ldots, \mathrm{PP}_{k}$.

Remarque 2 : Le problème PP peut également se poser comme la détermination d'une clique de poids maximal dans le graphe $\bar{G}$ (graphe complémentaire de $G$ ). Si $\bar{G}$ admet $k$ composantes connexes on recherchera une clique de poids maximal dans chacune des $k$ composantes et la clique obtenue ayant le plus grand poids est évidemment la clique de poids maximal de $\bar{G}$.

Exemple 1 ([12], p. 10) :

minimiser $z=30 x_{1}+49 x_{2}+51 x_{3}+52 x_{4}+52 x_{5}+55 x_{6}$

$$
+67 x_{7}+67 x_{8}+70 x_{9}+81 x_{10}+81 x_{11}+83 x_{12} \text {, }
$$

sous les contraintes $(1,2,3,4,8,9),(1,2,3,6,7,10),(1,4,5,6,8,11),(2,5,7,8,9$, $12),(3,4,9,10,11,12),(5,6,7,10,11,12)$ où $(1,2,3,4,8,9)$, par exemple, correspond à la contrainte $x_{1}+x_{2}+x_{3}+x_{4}+x_{8}+x_{9}=1$.

Le graphe $\bar{G}$ associé à ce problème est donné par la figure 1 .

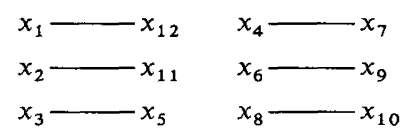

Figure 1

Il est évident, sur cet exemple, que la solution est :

$$
x_{3}=x_{5}=1, \quad x_{1}=x_{2}=x_{4}=x_{6}=x_{7}=x_{8}=x_{9}=x_{10}=x_{11}=x_{12}=0
$$

ce qui donne à $z$ la valeur 103 .

\section{EVALUATION PAR EXCÈS DE LA SOLUTION}

Pour obtenir une bonne évaluation par excès de la solution du problème PP nous cherchons à déterminer, dans le graphe associé à ce problème, un ensemble stable dont le poids soit aussi près que possible de celui d'un ensemble stable de poids maximal. Si $S$ est l'ensemble stable déterminé, une évaluation par excès de la solution du problème PP est alors : $m . L-P(S)$.

Nous avons testé un certain nombre d'heuristiques et nous présentons ici les deux que nous avons retenues comme étant les plus efficaces, l'heuristique 1 s'adaptant particulièrement bien aux problèmes de partitionnement peu contraints. 
Sur tous les exemples de la littérature que nous avons étudiés ces deux heuristiques donnent la solution du problème PP. Elles donnent également, comme "sous-produit " d'autres solutions admissibles du problème $A x=e$ souvent proches de la solution optimale. Il est extrêmement rapide de déterminer si un ensemble stable $S$ correspond à une solution de $A x=e$; une condition nécessaire et suffisante est, en effet, que son poids soit supérieur à $(m-l) L$ [1]. Dans ce cas, on associe à l'ensemble stable $S$ déterminé une solution admissible du problème de partitionnement :

$$
x_{j}=1 \quad \Leftrightarrow \quad x_{j} \in S .
$$

Soit $G=(X, V)$ avec $X=\left\{x_{1}, \ldots, x_{n}\right\}$ le graphe sur lequel on cherche à déterminer un « bon ensemble stable». Le meilleur ensemble stable obtenu est noté $S_{0}$.

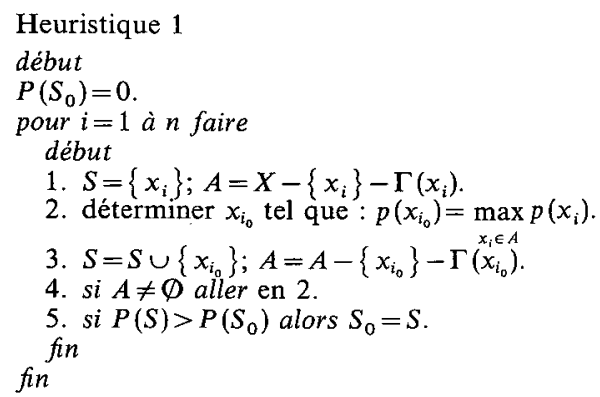

Heuristique 2

Elle se déroule exactement de la même façon que l'heuristique 1 excepté en ce qui concerne le point 2. qu'il faut remplacer par :

2. déterminer $x_{i_{0}}$ tel que :

$$
p\left(x_{i_{0}}\right) /\left|\Gamma\left(x_{i_{0}}\right)\right|=\max _{x_{i} \in A} p\left(x_{i}\right) /\left|\Gamma\left(x_{i}\right)\right| .
$$

\section{IMPLICATIONS}

Soit $z=z_{d}+\sum_{j=1}^{n} c_{j}^{\prime} x_{j}$ une réduction de la fonction économique et $z_{e}$ l'évaluation par excès de la solution obtenue par l'une des heuristiques précédentes. La solution du problème PP étant inférieure ou égale à $z_{e}$, nous chercherons une solution de valeur inférieure en simplifiant le problème $\mathrm{PP}$ au moyen des implications suivantes :

$$
-\forall j \in\{1, \ldots, n\}, z_{d}+c_{j}^{\prime} \geqq z_{e} \Rightarrow x_{j}=0 ;
$$


- les implications classiques dues aux contraintes $A x=e$ (cf. [5], p. 302).

Soit $x^{*}=\left(x_{1}^{*}, \ldots, x_{n}^{*}\right)$ la solution du problème PP (de valeur $\left.z^{*}\right)$. La prise en compte des différentes implications peut aboutir aux trois situations suivantes :

- contradiction rencontrée au cours de la simplification. On en déduit, alors, que $z^{*}=z_{e}$ et $x^{*}$ s'obtient immédiatement à partir de $S_{0}$;

- les simplifications s'effectuent normalement mais, finalement, toutes les variables sont fixées à 0 ou 1 ce qui détermine une solution, $x_{0}$, de $A x=e$. Deux cas peuvent se présenter :

$$
\begin{array}{lll}
z_{0} \geqq z_{e} & \text { alors } & z^{*}=z_{e}, \\
z_{0}<z_{e} & \text { alors } & z^{*}=z_{0} ;
\end{array}
$$

- les simplifications s'effectuent normalement et l'on aboutit à un problème de partitionnement plus simple sur lequel on peut effectuer une nouvelle réduction tout en tenant compte, dans le calcul de $z_{d}$, des variables déjà fixées à 1 .

\section{EXEMPLES}

Exemple 2 ([5], p. 315) :

$$
\text { minimiser } z=3 x_{1}+7 x_{2}+5 x_{3}+8 x_{4}+10 x_{5}+4 x_{6}+6 x_{7}+9 x_{8},
$$

sous les contraintes $(1,2),(3,4,5),(5,6,7),(7,8),(2,4,6)$.

La réduction de la fonction économique fournit, en choisissant successivement les contraintes $(7,8),(1,2),(3,4,5)$ et $(2,4,6)$ :

$$
z=17+x_{2}+5 x_{5}+x_{6}+3 x_{8} .
$$

Évaluation par excès.

Pour simplifier les calculs manuels on considèrera que la fonction à minimiser est $z^{\prime}=x_{2}+5 x_{5}+x_{6}+3 x_{8}$.

En prenant $L=11$ on trouve, par l'heuristique 1, les ensembles stables maximaux suivants :

$$
\begin{gathered}
\left\{x_{1}, x_{4}, x_{7}\right\}, \quad\left\{x_{2}, x_{3}, x_{7}\right\}, \quad\left\{x_{2}, x_{5}, x_{8}\right\}, \\
\left\{x_{1}, x_{3}, x_{6}, x_{8}\right\} \quad \text { et } \quad\left\{x_{1}, x_{4}, x_{8}\right\},
\end{gathered}
$$

dont les poids respectifs sont $55,54,46,51$ et 41 . Le poids des quatre premiers est supérieur à 44; ils correspondent donc à des solutions admissibles de $A x=e$. Prenons celui de plus fort poids : $\left\{x_{1}, x_{4}, x_{7}\right\}$. On en déduit que la valeur de $z^{*}$ est inférieure à 17 . 


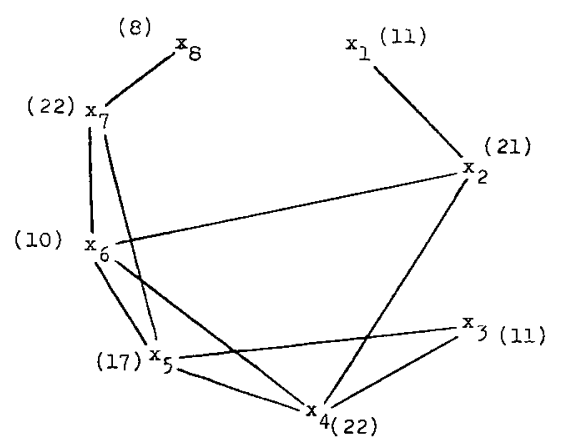

Figure 2. - Le poids de chaque sommet est indiqué entre parenthèses.

ImPLiCATIONS : Il apparaît immédiatement que la solution du problème PP est $x_{1}=x_{4}=x_{7}=1$.

Exemple 3 ([12], p. 11) :

minimiser $z=x_{1}+2 x_{2}+3 x_{3}+4 x_{4}+\ldots+18 x_{18}+19 x_{19}+20 x_{20}$,

sous les contraintes $(1,2,5,7,13),(2,7,14,18),(3,9,10,11),(4,6,8,12),(4,9,11$, $13,19),(5,10,15),(5,16,20),(8,14,15,17)$.

Réduction de la fonction économique : en appliquant les critères de réduction, on est amené à choisir successivement les contraintes $n^{\text {os }} 7,8,5,3$ et 2 et l'on obtient :

$$
\begin{aligned}
z=22+x_{1}+6 x_{6}+7 x_{7}+2 x_{9} & +5 x_{10}+4 x_{11}+12 x_{12}+9 x_{13}+4 x_{14} \\
& +7 x_{15}+11 x_{16}+9 x_{17}+16 x_{18}+15 x_{19}+15 x_{20} .
\end{aligned}
$$

Évaluation par excès.

L'ensemble stable de plus fort poids trouvé grâce à l'heuristique 1 est :

$$
S=\left\{x_{3}, x_{4}, x_{5}, x_{14}\right\} .
$$

Son poids est $1196(L=150)$. On en déduit que la valeur de $z^{*}$ est inférieure ou égale à 26 .

IMPLICATIONS : On assigne à 0 les variables de coût réduit supérieur ou égal à $26-22=4:$

$$
x_{6}=x_{7}=x_{10}=x_{11}=x_{12}=x_{13}=x_{14}=x_{15}=x_{16}=x_{17}=x_{18}=x_{19}=x_{20}=0 \text {, }
$$

puis $x_{2}=x_{5}=x_{8}=1$ ce qui viole la première contrainte. La solution optimale est donc : $x_{5}=x_{4}=x_{5}=x_{14}=1$; sa valeur est 26 . 
Exemple 4 ([9], p. 10) :

$$
\text { minimiser } \begin{aligned}
z & =x_{1}+x_{2}+x_{3}+x_{4}+x_{5}+x_{6}+x_{7}+x_{8} \\
& +2 x_{9}+2 x_{10}+2 x_{11}+2 x_{12}+2 x_{13}+2 x_{14}+2 x_{15} \\
+ & 3 x_{16}+3 x_{17}+3 x_{18}+3 x_{19}+4 x_{20}+4 x_{21}+4 x_{22}+4 x_{23} \\
+ & 5 x_{24}+5 x_{25}+5 x_{26}+6 x_{27}+6 x_{28}+6 x_{29}+7 x_{30}+8 x_{31}+9 x_{32},
\end{aligned}
$$

sous les contraintes $(1,9,19,22,25,28,32),(2,12,15,19,21,23,27,29,30,31$, $32),(3,10,19,24,26,30,32),(4,21,25,28,32),(5,11,16,22,23,27,31),(6,20$, $24,26,30,32),(7,12,17,25,30,31),(8,17,20,22,23,27,32),(8,13,14,26,29,30$, $31),(10,21,25,31,32),(14,15,18,23,24,27,30,32),(18,19,22,24,26,29,31)$, $(11,20,25,28,30,32),(16,19,23,31),(9,18,26,28,31,32)$.

Réduction de la fonction économique :

$$
\begin{aligned}
z=13+x_{1}+x_{2}+x_{3}+x_{5} & +x_{7}+2 x_{12}+x_{13}+x_{15}+3 x_{17}+x_{20} \\
& +x_{21}+4 x_{22}+x_{24}+x_{26}+5 x_{27}+x_{28}+5 x_{29}+2 x_{30} .
\end{aligned}
$$

Évaluation par excès.

L'ensemble stable de plus fort poids trouvé est $S=\left\{x_{23}, x_{25}, x_{26}\right\}$; son poids est $509(L=34)$. La valeur de $z$ est donc inférieure ou égale à 14 .

IMPLICATIONS : Toutes les variables de coût réduit non nul sont égales à 0 . Les contraintes deviennent alors : $(9,19,25,32),(19,23,32),(25,32),(11,16,23,31)$, $(6,32),(25,31),(8,23,32),(8,14,31),(10,25,31,32),(14,18,23,32),(18,19,31)$, $(11,25,32),(16,19,23,31),(9,18,31,32)$.

La simplification de ces contraintes conduit à une contradiction. Cela signifie que le système d'équations ainsi défini n'a pas de solutions.

Une solution optimale du problème est donc $x_{23}=x_{25}=x_{26}=1$ et sa valeur est 14 .

\section{QUELQUES REMARQUES}

Dans de nombreux cas, la méthode proposée permet de résoudre le problème de partitionnement. Beaucoup d'algorithmes permettent de trouver assez rapidement une bonne solution ou même la solution optimale mais la vérification de l'optimalité exige un temps très important ( $c f$. [2], p. 57). Dans la méthode que nous présentons et, pour tous les exemples étudiés, cette vérification est immédiate.

Cette méthode qui ne résout pas, à coup sûr, le problème de partitionnement permet, non seulement, de réduire la taille du problème mais peut aussi être 
facilement intégrée dans une procédure arborescente visant à l'optimum. Elle permet, alors, de réduire considérablement la taille de l'arborescence développée.

Soit $H_{v}$ l'ensemble des variables égales à 1 dans au moins une solution admissible de $A x=e$ dont la valeur est strictement inférieure à $v$. Il est évident que quelle que soit la réduction effectuée sur la fonction économique, si $z_{e}$ est l'évaluation, par excès, obtenue, on ne pourra avoir comme implication : $x_{j}=0$ si $x_{j}$ appartient à $H_{z_{e}}$. Le plus mauvais cas, pour notre méthode, serait donc que l'évaluation $z_{0}$ ne soit pas la valeur de la solution optimale et que les variables égales à 1 dans au moins une solution admissible, de valeur inférieure à $z_{e}$, soient très nombreuses.

\section{AMÉLIORATIONS POSSIBLES DE LA MÉTHODE}

Dans le cas où l'on parvient à un problème PP plus simple que le problème initial mais dans lequel la méthode ne permet plus d'effectuer de simplifications deux possibilités s'offrent, alors, pour obtenir de nouvelles implications :

(i) effectuer des réductions différentes de la fonction économique tout en essayant de conserver une bonne évaluation par défaut. Le nombre de variables susceptibles d'être fixées à 0 est ainsi augmenté (des réductions différentes sont obtenues en modifiant l'ordre dans lequel on choisit les contraintes).

Exemple 5 ([6], p. 851) :

$$
\text { minimiser } \begin{aligned}
z=18 x_{1}+22 x_{2}+14 x_{3}+36 x_{4}+17 x_{5} & +14 x_{6} \\
& +8 x_{7}+24 x_{8}+14 x_{9}+7 x_{10},
\end{aligned}
$$

sous les contraintes $(3,5),(4,5,6),(2,4,8,9),(3,4,8,10),(1,2,7,9),(1,2,4,6,8)$.

Gondran et Laurière proposent dans [7], p. 30, les deux réductions :

$$
z=39+10 x_{1}+11 x_{4}+3 x_{6}+8 x_{7}+2 x_{8}+7 x_{10}
$$

et

$$
z=39+19 x_{4}+x_{6}+10 x_{8}+2 x_{9}+7 x_{10} .
$$

Dans ce problème, l'évaluation par excès obtenue est 42 . Pour la recherche d'une solution de valeur inférieure à 42 , la deuxième réduction, par exemple, permet de fixer la variable $x_{8}$ à 0 alors que la première ne le permet pas.

vol. $15, \mathrm{n}^{\circ} 2$, mai 1981 
(ii) essayer d'augmenter, au maximum, un coût réduit sans modifier l'évaluation par défaut, ce qui permet, comme précédemment, d'obtenir de nouvelles implications du type : $z_{d}+c_{j}^{\prime} \geqq z_{e} \Rightarrow x_{j}=0$.

Le principe est le suivant :

soit $z=z_{d}+\sum_{j=1}^{n} c_{j}^{\prime} x_{j}$ une expression réduite de la fonction économique. Considérons deux contraintes quelconques du problème $\mathrm{PP}$ :

$$
\sum_{j=1}^{n} a_{i_{1} j} x_{j}=1 \quad \text { et } \quad \sum_{j=1}^{n} a_{i_{2} j} x_{j}=1
$$

Appelons $\Delta$ le plus petit coût réduit des variables qui apparaissent dans la deuxième contrainte mais non dans la première. On obtient une nouvelle réduction de la fonction économique dans laquelle le coefficient de chaque variable est positif ou nul :

$$
z=z_{d}+\sum_{j \in J_{i_{1}}}\left(c_{j}^{\prime}+\Delta\right) x_{j}+\sum_{j \in J_{i_{2}}}\left(c_{j}^{\prime}-\Delta\right) x_{j}+\sum_{j \in J-\left(J_{i_{1}} \cup J_{i_{2}}\right)} c_{j}^{\prime} x_{j}
$$

où

$$
J_{i_{1}}=\left\{j \mid a_{i_{j}}=1\right\} \quad \text { et } \quad J_{i_{2}}=\left\{j \mid a_{i_{2} j}=1\right\} .
$$

Cette transformation a permis d'augmenter le coût réduit des variables qui apparaissent dans la première contrainte mais non dans la seconde.

Présentons maintenant un algorithme qui permet d'augmenter les coûts réduits. Pour une variable $x_{j}$ le plus grand coût réduit obtenu est noté $c_{j}^{\prime}$ max (cela signifie qu'il existe une réduction de la fonction économique où le coût réduit de $x_{j}$ est $c_{j}^{\prime} \max$ ).

\section{Notations :}

$$
\begin{gathered}
I=\{1, \ldots, m\} \quad \text { et } \quad J=\{1, \ldots, n\} \\
K_{j}=\left\{k \mid a_{k j}=1\right\} \quad \text { et } \quad \bar{K}_{j}=I-K_{j} ; \\
J_{i}=\left\{j \mid a_{i j}=1\right\} \\
H_{i}^{k}=J_{i}-\left(J_{k} \cap J_{i}\right) \\
\Delta_{i}^{k}=\min _{c_{j}}
\end{gathered}
$$

R.A.I.R.O. Recherche opérationnelle/Operations Research 


\section{ALGORITHME :}

pour $j=1$ à $n$ faire $c_{j}^{\prime} \max =c_{j}^{\prime}$

pour $j=1$ à $n$ faire

début

considérer la fonction économique sous sa forme réduite initiale $z=z_{d}+\sum_{j=1}^{n} c_{j}^{\prime} x_{j}$

pour tout $k \in K_{j}$ faire

pour tout $i \in \bar{K}_{\text {, faire }}$

détut

calculer $\Delta_{i}^{k}$

calculer $z=z+\Delta_{i}^{k}\left(\sum_{j=1}^{n} a_{k, j} x_{j}-\sum_{j=1}^{n} a_{i j} x_{j}\right)$ (commentaire : le coefficient de $x_{j}$ augmente de $\Delta_{i}^{k}$;

on appellera toujours $c_{l}^{\prime}$ le coefficient de $x_{l}$ dans la nouvelle expression de z.)

pour $l \in H_{i}^{k}$ faire si $c_{l}^{\prime}>c_{l}^{\prime} \max$ alors $c_{l}^{\prime} \max =c_{l}^{\prime}$

fin fin

Remarques : Soit $v_{i}$ la plus petite valeur des solutions admissibles de $A x=e$ dans lesquelles $x_{i}=1$. Il est évident que, pour toute expression réduite de la fonction économique, l'inégalité suivante est vérifiée :

$$
\forall i, \quad c_{i}^{\prime} \leqq v_{i}-z_{d} .
$$

L'heuristique présentée permet très souvent de parvenir à l'égalité.

On peut essayer d'augmenter, au maximum, le coût réduit d'une variable à partir de l'expression initiale de la fonction économique sans avoir procédé à une première réduction.

\section{Amélioration de l'évaluation par défaut}

Le fait de chercher à augmenter le coût réduit de chaque variable permet d'obtenir, en plus des implications citées précédemment, une excellente évaluation par défaut de la solution du problème PP, souvent meilleure que la solution du problème PP en continu. On sait que la connaissance d'une bonne évaluation par défaut est très intéressante pour accélérer le déroulement d'une procédure arborescente.

Soit $c_{j}^{\prime}$ max le coût réduit maximal obtenu pour la variable $x_{j}$; une évaluation par défaut de la solution du problème PP est alors :

Exemple 6 :

$$
z_{d}^{\prime}=z_{d}+\max _{i \in I}\left(\min _{j \in J_{i}} c_{j}^{\prime} \max \right) .
$$

$$
\text { minimiser } z=x_{1}+x_{2}+5 x_{3}+5 x_{4}+5 x_{5}+5 x_{6} \text {, }
$$

sous les contraintes $(1,2,4),(1,3,5)$ et $(2,3,6)$. 
La fonction économique réduite est :

$$
z=2+3 x_{3}+4 x_{4}+4 x_{5}+4 x_{6} .
$$

En appliquant l'algorithme précédent on obtient :

$$
\begin{array}{lll}
c_{1} \max =3, & c_{2} \max =3, & c_{3} \max =3, \\
c_{4} \max =7, & c_{5} \max =4, & c_{6} \max =4 .
\end{array}
$$

Une évaluation par défaut de la solution de ce problème est donc 5 .

Si l'on s'intéresse au problème précédent, en continu, une solution évidente est :

$$
x_{1}=x_{2}=x_{3}=1 / 2, \quad x_{4}=x_{5}=x_{6}=0 \quad \text { et } \quad z=3,5 .
$$

L'évaluation par défaut obtenue par la résolution du programme linéaire continu est donc, dans cet exemple, moins bonne que celle déterminée par notre méthode.

\section{RESULTATS}

Nous avons programmé, en ALGOL W, les deux heuristiques du paragraphe 2 qui permettent d'obtenir une évaluation par excès de la solution du problème de partitionnement.

Dans presque tous les cas que nous avons examinés, l'heuristique 2 s'est révélée supérieure à l'heuristique 1 . Nous donnons, dans le tableau I les resultats obtenus, par l'heuristique 2 , pour dix problemes de partitionnement de 100 variables, engendrés aléatoirement mais ayant au moins une solution admissible.

$m$ désigne le nombre de contraintes du problème, $z_{e}$ l'évaluation par excès obtenue et $z^{*}$ la valeur de la solution.

TABLEAU I

\begin{tabular}{|c|c|c|c|c|c|}
\hline$m$ & $z_{\boldsymbol{e}}$ & $z^{*}$ & $m$ & $z_{c}$ & $z^{*}$ \\
\hline 10 & 44 & 24 & 35 & 70 & 65 \\
20 & 92 & 76 & 35 & 114 & 100 \\
20 & 130 & 80 & 40 & 330 & 245 \\
25 & 223 & 223 & 50 & 211 & 211 \\
30 & 143 & 115 & 57 & 648 & 648 \\
\hline
\end{tabular}

R.A.I.R.O. Recherche opérationnelle/Operations Research 
REMARQue : Dans tous les cas traités, cette heuristique a permis d'obtenir une solution admissible du problème.

Nous avons également programmé la méthode proposée par Gondran et Laurière pour réduire la fonction économique (ce qui donne une évaluation par défaut, $z_{d}$, de la solution) suivie de la méthode que nous proposons au paragraphe 8 pour augmenter au maximum les coûts réduits ce qui permet, en particulier, d'obtenir une autre évaluation par défaut $z_{d}^{\prime}$.

Le tableau II présente les résultats obtenus concernant ces deux évaluations par défaut. (ll s'agit toujours de problèmes de partitionnement, de 100 variables, engendrés aléatoirement.)

TABleau II

\begin{tabular}{|c|c|c|c||c|c|c|c|}
\hline$m$ & $z_{d}$ & $z_{d}^{\prime}$ & $z^{*}$ & $m$ & $z_{d}$ & $z_{d}^{\prime}$ & $z^{*}$ \\
\hline 10 & 17 & 20 & 24 \\
20 & 45 & 52 & 76 \\
20 & 58 & 72 & 80 \\
40 & 109 & 130 & 234 & 131 & 149 & 245 \\
50 & 88 & 116 & 211 \\
50 & 26 & 61 & 281 \\
\hline
\end{tabular}

On peut constater que, dans tous les cas examinés, la méthode présentée au paragraphe 8 permet d'améliorer sensiblement l'évaluation par défaut obtenue.

Enfin, nous avons programmé, d'une part, l'algorithme de Gondran et Laurière et, d'autre part, ce même algorithme après y avoir apporté les modifications suivantes :

(a) calcul, en début d'algorithme, d'une évaluation par excès, $z_{e}$, par l'heuristique 2 du paragraphe 4;

(b) en chaque nœud de l'arborescence, on tient compte des implications $z_{d}+c_{j}^{\prime} \geqq z_{e} \Rightarrow x_{j}^{\prime}=0$, puis on augmente les coûts réduits des variables non encore fixées, par l'algorithme du paragraphe 8 , ce qui fournit une nouvelle évaluation par défaut dont on tient compte, ainsi que de nouvelles implications.

Dans tous les cas que nous avons étudiés, les modifications $(a)$ et $(b)$ permettent de réduire considérablement la taille de l'arborescence développée. Nous donnons dans le tableau III les premiers résultats obtenus (problèmes de partitionnement de 100 variables engendrés aléatoirement). $N_{1}$ désigne le nombre de sommets de l'arborescence développée par notre méthode et $N_{2}$ le nombre de sommets de l'arborescence développée par l'algorithme de Gondran et Laurière.

vol. $15, \mathrm{n}^{\circ} 2$, mai 1981 
TABLEAU III

\begin{tabular}{|c|c|c|c||c|c|c|c|}
\hline$m$ & $N_{1}$ & $N_{2}$ & $z^{*}$ & $m$ & $N_{1}$ & $N_{2}$ & $z^{*}$ \\
\hline 10 & 8 & 49 & 24 & 20 & 17 & 28 & 76 \\
20 & 37 & - & 80 & 50 & 12 & 16 & 211 \\
40 & 63 & 237 & 245 & & & & \\
\hline
\end{tabular}

- La solution de ce problème n'a pas été déterminée dans le temps qui nous était accordé.

\section{REMERCIEMENTS}

Je remercie MM. M. Ben Cheikh et O. Haligon pour l'aide qu'ils m'ont apportée dans cette étude.

\section{BIBLIOGRAPHIE}

1. A. Billionnet, Transformation du probleme de partitionnement en un problème d'ensemble stable de poids maximal, R.A.I.R.O., vol. V-3, 1978, p. 319-323.

2. J. Delorme et E. Heurgon, Problèmes de partitionnement : exploration arborescente ou méthode de troncatures?, R.A.I.R.O., vol. V-2, 1975, p. 53-65.

3. R. FAURE, Polycopié de l'Institut de Programmation : graphes et applications, Paris, 1969.

4. J. FreHeL, Régularisation dans les problèmes combinatoires, problèmes de tournées, de partition et de recouvrement, Communication du 4 décembre 1975 à la réunion du groupe combinatoire de l'A.F.C.E.T.

5. R. S. Garfinkel et G. L. Nemhauser, Integer Programming, chap. 8, John Wiley and Sons, 1972.

6. R. S. Garfinkel et G. L. Nemhauser, The Set Partitionning Problem: Set Covering with Equality Constraints, Opns. Res., vol. 17, 1969, p. 848 à 856.

7. M. Gondran et J. L. LAURIÈRE, Un algorithme pour le problème de partitionnement, R.A.I.R.O., vol. V-1, 1974, p. 27 à 40.

8. E. Heurgon, Un problème de recouvrement: l'habillage des horaires d'une ligne d'autobus, R.A.I.R.O., vol. V-1, 1972, p. 13 à 29.

9. C. E. Lemke, H. M. Salkin et K. Spielberg, Set Covering by Single Branch Enumeration with Linear Programming Subproblems, Oper. Res., vol. 19, 1971.

10. J. F. PIERCE, Application of Combinatorial Programming to a Class of All-Zero-One Integer Programming Problems, Man. Sc., vol. 15, 1968, p. 191 à 209.

11. J. F. PIERCE et J. F. LASKY, Improved Combinatorial Programming Algorithms for a Class of All-Zero-One Integer Programming Problems, Man. Sc., vol. 19, n 5, 1973, p. 528 à 543.

12. K. Vo-K $\mathrm{HAC}$, Utilisation des coûts pondérés et des variables bivalentes dans les problèmes des tournées : sectorisation sous contraintes nombreuses, R.A.I.R.O., vol. V-2, 1972, p. 3 à 20. 